\title{
Conformal Carroll groups and BMS symmetry
}

\author{
C. Duval ${ }^{1 *}$, G. W. Gibbons ${ }^{2,3,4 \dagger}$, P. A. Horvathy ${ }^{3 \ddagger}$, \\ ${ }^{1}$ Centre de Physique Théorique, Marseille, France \\ ${ }^{2}$ D.A.M.T.P., Cambridge University, U.K. \\ ${ }^{3}$ Laboratoire de Mathématiques et de Physique Théorique, Université de Tours, France \\ ${ }^{4}$ LE STUDIUM, Loire Valley Institute for Advanced Studies, Tours and Orleans France
}

(Dated: April 4, 2014)

\begin{abstract}
The Bondi-Metzner-Sachs (BMS) group is shown to be the conformal extension of Lévy-Leblond's "Carroll" group. Further extension to the Newman-Unti (NU) group is also discussed in the Carroll framework.

PACS numbers: 04.20.-q Classical general relativity; 04.20.Ha Asymptotic structure; 02.40.-k Geometry, differential geometry, and topology; 02.20.Sv Lie algebras of Lie groups; 02.20.Tw Infinite-dimensional Lie groups;
\end{abstract}

\section{INTRODUCTION}

There has recently been a resurgence of interest in both the Bondi-Metzner-Sachs (BMS) group [1-4]) and, independently, in Lévy-Leblond's "Carroll” group [5-7].

The BMS group arose as the asymptotic symmetry group of a four dimensional asymptotically flat spacetime repre-

*Aix-Marseille Université, CNRS, CPT, UMR 7332, 13288 Marseille, France. Université de Toulon, CNRS, CPT, UMR 7332, 83957 La Garde, France. mailto:duval@cpt.univ-mrs.fr

${ }^{\dagger}$ mailto:G.W.Gibbons@damtp.cam.ac.uk

${ }_{\ddagger}^{\ddagger}$ mailto:horvathy@lmpt.univ-tours.fr senting an isolated time-dependent system emitting gravitational radiation [1]. It had been anticipated, as is the case for asymptotically flat time independent isolated systems, that the asymptotic symmetry group would be the Poincaré group and it came as a surprise that in the presence of gravitational radiation it is impossible to isolate a unique asymptotic Poincaré group, but rather the asymptotic symmetries constitute an infinite-dimensional group which contains many copies of the Poincaré group, none of which being invariant.

The BMS group may be thought of as acting on future (or past) null infinity $\mathcal{I}^{ \pm}$. The 
latter are null hypersurfaces contained in the conformal boundary of an asymptotically flat spacetime. Topologically $\mathcal{I}^{ \pm} \equiv S^{2} \times \mathbb{R}$, where the $S^{2}$ factor corresponds to the 2-sphere of asymptotic directions and the $\mathbb{R}$ factor to retarded (advanced) time. Thus local charts $\theta, \phi, u$ or $\theta, \phi, v$ may be introduced, where $\theta, \phi$ parametrize the null generators and $u, v$ are affine parameters along the null generators. From now on we shall consider only $\mathcal{I}^{+}$since the story for $\mathcal{I}^{-}$is identical.

Let us recall that $\mathcal{I}^{+}$admits a degenerate conformal structure for which we may take a representative metric of the form $d s^{2}=$ $0 \times d u^{2}+d \theta^{2}+\sin ^{2} \theta d \phi^{2}$, where the nonvanishing summand is the standard round metric on the unit 2-sphere which we wish to think of as the Riemann sphere $\mathbb{C} \cup\{\infty\}$. To this end we introduce stereographic coordinates $\zeta=e^{i \phi} \cot (\theta / 2)$ in terms of which $d s^{2}=4(1+\zeta \bar{\zeta})^{-2} d \zeta d \bar{\zeta}$. The identity component of the Lorentz group is isomorphic to $\operatorname{PSL}(2, \mathbb{C})=\operatorname{SL}(2, \mathbb{C}) / \mathbb{Z}_{2}$ and acts as conformal transformations of the Riemann sphere. Specifically, if $a, b, c, d \in \mathbb{C}$ are such that $a d-b c=1$, then for

$$
\zeta^{\prime}=\varphi(\zeta)=\frac{a \zeta+b}{c \zeta+d}
$$

we have

$$
\frac{d \zeta^{\prime} d \bar{\zeta}^{\prime}}{(1+\tilde{\zeta} \bar{\zeta})^{2}}=\Omega^{2}(\zeta, \bar{\zeta}) \frac{d \zeta d \bar{\zeta}}{(1+\zeta \bar{\zeta})^{2}}
$$

where

$$
\Omega(\zeta, \bar{\zeta})=\frac{1+\zeta \bar{\zeta}}{|a \zeta+b|^{2}+|c \zeta+d|^{2}} .
$$

The infinite-dimensional abelian group $\mathcal{T}$ of super-translations acts on the 2-sphere sections (known as "cuts") of the product as $u \rightarrow u^{\prime}=u+\alpha(\zeta, \bar{\zeta})$ preserving the conformal structure, where $\alpha(\zeta, \bar{\zeta})$ is a smooth real valued function on the Riemann sphere which transforms as a scalar of weight 1 under conformal transformations of $S^{2}[8,9]$. The standard BMS group is thus the semi-direct product of $\operatorname{PSL}(2, \mathbb{C})$ with $\mathcal{T} \equiv C^{\infty}\left(S^{2}, \mathbb{R}\right)$ and acts on $\mathcal{I}^{+}$as $(\zeta, u) \mapsto\left(\zeta^{\prime}, u^{\prime}\right)$ with $\zeta^{\prime}$ as in Eq. (I.1), and

$$
u^{\prime}=\Omega(\zeta, \bar{\zeta})[u+\alpha(\zeta, \bar{\zeta})]
$$

If $\alpha$ is expanded in spherical harmonics and only the $l=0$ and $l=1$ terms retained, one obtains a closed subgroup isomorphic with the Poincaré group. Now, the sum of the $l=0$ and $l=1$ representations of $\mathrm{SO}(3)$ transform as the $D^{\left(\frac{1}{2} \frac{1}{2}\right)}$ representation of $\operatorname{PSL}(2, \mathbb{C})$, i.e., as the defining representation of the Lorentz group and as a scalar of weight 1 under conformal transformations. However the Poincaré subgroup so defined is not an invariant subgroup [10]. In that case the generalized BMS group (and also its Lie algebra of vector fields) will be much larger than the Poincaré subgroup defined above.

Rather weaker versions of the BMS group 
may be defined. For example, the NewmanUnti (NU) group is defined by choosing $a, b, c, d \in \mathbb{C}$ such that $a d-b c=1$, and set

$$
\zeta^{\prime}=\frac{a \zeta+b}{c \zeta+d}, \quad u^{\prime}=f(\zeta, \bar{\zeta}, u)
$$

where $f$ must decrease with $u$ at fixed $\zeta$ [10]. The NU group preserves the conformal geometry, but not what is called the strong conformal geometry [10]. There is also an intermediate version, for which

$$
f(\zeta, \bar{\zeta}, u)=\beta(\zeta, \bar{\zeta})[u+\alpha(\zeta, \bar{\zeta})]
$$

Taking $\beta(\zeta, \bar{\zeta})=\Omega(\zeta, \bar{\zeta})$ gives the general element of the BMS group.

The obvious generalization is to replace $S^{2}$ by $S^{d}$ and $\mathrm{O}(3,1)$ by $\mathrm{O}(d+1,1)[11]$, but since the asymptotics of solutions of the Einstein's equations in greater than four spacetime dimensions differ somewhat from the four-dimensional case [12], its physical relevance remains unclear. More generally, one may clearly consider the group obtained by replacing the Riemann sphere by any closed Riemannian manifold $(\Sigma, \widehat{\mathrm{g}})$. In general this will have no proper conformal isometries and so one gets the semi-direct product of the isometry group of $(\Sigma, \widehat{\mathrm{g}})$ with $C^{\infty}(\Sigma, \mathbb{R})$. If $\Sigma$ is non-compact, for example the Euclidean plane $\mathbb{E}^{2} \equiv \mathbb{C}$, then if one is unconcerned about global issues, in a formal sense any holomorphic map is conformal. Similarly for the cylinder $\mathbb{E}^{2} \backslash\{0\} \equiv \mathbb{C} \backslash\{0\} \equiv S^{1} \times \mathbb{R}$.
In that case the generalized BMS group and its Lie algebra will be much larger than that defined above. It is this type of infinitedimensional extension of the standard BMS group which has figured in much of the current literature $[2,3]$.

Our purpose being to relate all these groups to some particular overgroups of the Carroll group, let us recall that the latter, originally introduced as an unusual contraction of the Poincaré group [5], is highlighted by that a "Carrollian boost" with parameter $\mathbf{b} \in \mathbb{R}^{3}$ transforms "Carrollian time" $u$ alone, according to

$$
\mathbf{x}^{\prime}=\mathbf{x}, \quad u^{\prime}=u-\mathbf{b} \cdot \mathbf{x}
$$

where $\mathbf{x} \in \mathbb{R}^{3}$, instead of the familiar Galilean action $\mathbf{x}^{\prime}=\mathbf{x}+\mathbf{b} t, t^{\prime}=t$ on non-relativistic spacetime.

The aim of the present Letter is thus to show that the BMS and NU groups can be understood as conformal symmetries, namely as conformal Carroll groups introduced in this paper, associated with Carroll manifolds $[6,7]$. Further extension to the Newman-Unti group [10] is also discussed.

\section{CARROLL GROUP AND MANI- FOLD}

In [7] a definition independent of relativity and group contraction has been put for- 
ward. It is based on defining a Carroll manifold analogous to a Newton-Cartan manifold $[7,13]$ as a $(d+1)$-dimensional manifold $C$ (for Carroll), endowed with a twicesymmetric covariant, positive tensor field, g, whose kernel is generated by a nowhere vanishing vector field $\xi$. The stronger definition proposed in Ref. [7] requires a Carroll manifold to carry, in addition, a symmetric affine connection, compatible with both $\mathrm{g}$ and $\xi$. The degeneracy of the "metric" g implies that the connection is not uniquely defined or may let alone exist, see Section IV below. In this Letter we stick essentially to our new and less restrictive definition given here.

(i) The standard Carroll structure is given by $C^{d+1}=\mathbb{R}^{d} \times \mathbb{R}$, with $\mathrm{g}=\delta_{A B} d x^{A} d x^{B}$, and $\xi=\partial / \partial u$, where $A, B=1, \ldots, d$ are spatial indices, and $u=x^{d+1}$ is the "Carrollian "time"-coordinate with dimension action/mass.

(ii) More general Carroll manifolds can be constructed out of hypersurfaces $\Sigma$ with Riemannian metric $\widehat{\mathrm{g}}$, namely as $C=\Sigma \times \mathbb{R}$ and $\mathrm{g}=\widehat{\mathrm{g}}_{A B}(x) d x^{A} d x^{B}$, and $\xi=\partial / \partial u$. In both cases, $C$ can also be endowed with a compatible connection, e.g., $\Gamma_{i j}^{k}=0$ for all $i, j, k=1, \ldots, d+1$ in case (i). The nonvanishing components of the Carroll connection of case (ii) being $\Gamma_{A B}^{C}=\widehat{\Gamma}_{A B}^{C}$ (the LeviCivita connection of $\widehat{\mathrm{g}}$ ), the $\Gamma_{A B}^{u}$ remaining arbitrary. Yet another important example will be studied in Section IV below.

\section{CONFORMAL}

CARROLL

\section{TRANSFORMATIONS}

Inspired by the definition of relativistic, and also non-relativistic [13], conformal transformations, we now introduce for, a given Carroll manifold, the conformal Carroll group of level $N, \operatorname{CCarr}_{N}(C, \mathrm{~g}, \xi)$, of those transformations which preserve the tensor field $\mathrm{g} \otimes \xi^{\otimes N}$ canonically associated with our Carroll structure, i.e., of all transformations, $a$, which satisfy

$$
a^{*} \mathrm{~g}=\Omega^{2} \mathrm{~g} \quad \& \quad a^{*} \xi=\Omega^{-2 / N} \xi
$$

for some positive function $\Omega$ on $C$ and positive integer $N$. The Lie algebra of infinitesimal conformal Carroll transformations, $\mathfrak{c c a r r}_{N}(C, \mathrm{~g}, \xi)$, is spanned, accordingly, by vector fields $X$ such that

$$
L_{X} \mathrm{~g}=\lambda \mathrm{g} \quad \& \quad L_{X} \xi=-\frac{\lambda}{N} \xi
$$

for some function $\lambda$ on $C$. In the flat case (i) our formulae yield [14]

$$
\begin{aligned}
X= & \left(\omega_{B}^{A} x^{B}+\gamma^{A}+\left(\chi-2 \kappa_{B} x^{B}\right) x^{A}\right. \\
& \left.+\kappa^{A} x_{B} x^{B}\right) \frac{\partial}{\partial x^{A}}+ \\
& \left(\frac{2}{N}\left(\chi-2 \kappa_{B} x^{B}\right) u+T(x)\right) \frac{\partial}{\partial u}
\end{aligned}
$$

where $\boldsymbol{\omega} \in \mathfrak{s o}(d), \boldsymbol{\gamma}, \boldsymbol{\kappa} \in \mathbb{R}^{d}$, and $\chi \in \mathbb{R}$, with $T \in C^{\infty}\left(\mathbb{R}^{d}, \mathbb{R}\right)$. In view of (III.1), the supertranslation $T$ in (III.3) has conformal weight 
$-2 / N$, and should therefore be regarded as a density with weight $\nu=-2 /(N d)$. Hence, $\mathfrak{c c a r r}_{N}(d+1)$ is the semi-direct product of the conformal Lie algebra $\mathfrak{s o}(d+1,1)$ with $\nu$-densities on $\mathbb{R}^{d}[16]$. Due to the degeneracy of the Carroll "metric", g, our conformal Carroll Lie algebras are infinite-dimensional, owing to super-translations represented by $T$. In view of (III.3), the quantity $z=2 / N$ is the associated dynamical exponent. The value $N=2$ is particularly interesting: space and "time" are then equally dilated so that the dynamical exponent is $z=1$ [17]. Moreover, in $d=1$ space dimension, interchanging position and time, $x \leftrightarrow s$ and renaming $s$ as $t$, our conformal Carroll algebra becomes precisely the Conformal Galilei algebra CGA [15], [13]. Note that the interchange also swaps Carrollian (I.7) and ordinary Galilean boosts.

Requiring $\Omega=1$, i.e., $\lambda=0$ in (III.2) would yield the "isometry group" of the Carrollian structure $(\mathrm{g}, \xi)$. It is infinitedimensional owing to the presence of supertranslations. Requiring, in addition, the preservation of a Carroll connection would reduce this to a finite-dimensional group; for, e.g., the flat Carroll structure (i) we get the usual Carroll group [5], denoted by $\operatorname{Carr}(d+1)$ in [7]. The Carroll Lie algebra, $\mathfrak{c a r r}(d+1)$, is spanned by the vector fields

$$
X=\left(\omega_{B}^{A} x^{B}+\gamma^{A}\right) \frac{\partial}{\partial x^{A}}+\left(\sigma-\beta_{A} x^{A}\right) \frac{\partial}{\partial u},
$$

where $\omega \in \mathfrak{s o}(d), \boldsymbol{\beta}, \boldsymbol{\gamma} \in \mathbb{R}^{d}$, and $\sigma \in \mathbb{R}$.

In case (ii), the general expression of a conformal Carroll vector field in $\mathfrak{c c a r r}_{N}(C, \mathrm{~g}, \xi)$ is

$$
X=Y+\left(\frac{\lambda}{N} u+T(x)\right) \frac{\partial}{\partial u},
$$

where $Y=Y^{A}(x) \partial / \partial x^{A}$ is a conformal vector field of $(\Sigma, \widehat{\mathrm{g}})$, i.e., such that $L_{Y} \widehat{\mathrm{g}}=\lambda \widehat{\mathrm{g}}$, hence with $\lambda=(2 / d) \widehat{\nabla}_{A} Y^{A}$, and $T$ is a real function on $\Sigma$. Integration of the vector field (III.5) readily yields the group action $(x, u) \mapsto\left(x^{\prime}, u^{\prime}\right)$, where

$$
x^{\prime}=\varphi(x), u^{\prime}=\Omega^{2 / N}(x)[u+\alpha(x)],
$$

with $\varphi \in \operatorname{Conf}(\Sigma, \widehat{g})$, and $\alpha \in C^{\infty}(\Sigma, \mathbb{R})$. Putting $a=(\varphi, \alpha)$, we readily find the group law of the conformal Carroll group of level $N$; if $a^{\prime \prime}=a^{\prime} a$, we end up with the group law

$$
\varphi^{\prime \prime}=\varphi^{\prime} \circ \varphi, \quad \alpha^{\prime \prime}=\Omega^{-2 / N} \varphi^{*} \alpha^{\prime}+\alpha .
$$

Notice, again, that supertranslations are actually densities of conformal weight $-2 / N$. The conformal Carroll transformations of $C=\Sigma \times \mathbb{R}$ belong therefore to the semidirect product of conformal transformations of $(\Sigma, \widehat{\mathrm{g}})$ with "supertranslations" of $\Sigma$,

$$
\operatorname{CCarr}_{N}(C, \mathrm{~g}, \xi) \equiv \operatorname{Conf}(\Sigma, \widehat{\mathrm{g}}) \ltimes \mathcal{T},
$$


where $\mathcal{T}$ is a shorthand for supertranslations (mathematically, $-2 /(N d)$-densities on $\Sigma$ ).

If, for example, $\Sigma=S^{1}$ and $\mathrm{g}=d \theta^{2}$, conformal Carroll transformations of level $N$ will be given by the semi-direct product of the conformal transformations of the circle, $\operatorname{Diff}\left(S^{1}\right)$, and supertranslations with weight $\nu=-2 / N$. They are generated by the vector fields

$$
X=Y(\theta) \frac{\partial}{\partial \theta}+\left(\frac{2}{N} Y^{\prime}(\theta) u+T(\theta)\right) \frac{\partial}{\partial u} .
$$

Considering instead $\Sigma=S^{2}$ endowed with its round metric allows us to conclude that the conformal Carroll transformations of level $N=2$ are the semi-direct product of the conformal group of $S^{2}$ with supertranslations,

$$
\operatorname{CCarr}_{2}\left(S^{2} \times \mathbb{R}, \mathrm{g}, \xi\right) \equiv \operatorname{PSL}(2, \mathbb{C}) \ltimes \mathcal{T}
$$

with $\mathcal{T}$ the $-1 / 2$ densities on the two-sphere. This is, precisely, the Bondi-Metzner-Sachs group BMS(4) [1] whose group law is given by (III.7). Its action on our Carroll manifold can be read off from (III.6) with $N=2$, and yielding Eqs (I.1) and (I.4).

Then, for $\Sigma=S^{d}$, the group $\operatorname{SL}(2, \mathbb{C})$ is simply replaced by (the neutral component of $) \mathrm{O}(d+1,1)$, and Carroll isometries readily identified with the semi-direct product of the orthogonal group $\mathrm{O}(d+1)$ with supertranslations - and is therefore still infinite-dimensional. We therefore claim that
$\mathrm{CCarr}_{2}(C, \mathrm{~g}, \xi) \equiv \operatorname{BMS}(d+2)$.

\section{THE LIGHT-CONE AS A CAR- ROLL MANIFOLD AND THE BMS GROUP}

To present our third example of a Carroll manifold, we deal with Minkowski spacetime $\mathbb{R}^{d+1,1}$ endowed with the metric $G=$ $\operatorname{diag}(1, \ldots, 1,-1)$, and look at the punctured future light cone, $C=\mathcal{I}^{+}$, of future pointing null vectors, described (non canonically) by those vectors $(\mathbf{z}, t) \in \mathbb{R}^{d+1} \times \mathbb{R}$ such that $t \equiv|\mathbf{z}|>0$. Let us then consider the symmetric tensor, g, inherited on $C$ from the Minkowski metric $G$. Using coordinates, and since $t>0$ can be viewed as a (global) radial coordinate on $C$, let the unit vector $\mathbf{x}=\mathbf{z} / t$ denote the direction of $\mathbf{z}$. Then $\mathrm{g}=t^{2}|d \mathbf{x}|^{2}=t^{2} \widehat{\mathrm{g}}$, where $\hat{g}$ is the usual round metric of $S^{d}$ (the directions of light rays). Let us insist that, the above decomposition of null vectors being non-canonical, "projecting" g to the "celestial sphere", $S^{d}$, only defines a conformal class, $[g]$, of metrics of the latter. The kernel of $g$ is seen to be spanned by the restriction, $\xi$, to $C$ of the Euler vector field of $\mathbb{R}^{d+1,1}$, namely $\xi=t \partial_{t}=\partial_{u}$, where $u=\log t$.

The light-cone $C=\mathcal{I}^{+}$is, hence, an intrinsically defined Carroll manifold. Can it be endowed with a compatible connection ? 
Crucially for us, the answer is no ! To see this, choose a coordinate system $\left(x^{A}, u\right)$ on $C$, such that $g_{A B}=e^{2 u} \hat{g}_{A B}, g_{A u}=0, g_{u u}=0$, $\xi^{A}=0, \xi^{u}=1$. Then a symmetric affine connection $\nabla$ on $C$ should satisfy both conditions $\left(\nabla_{u} g\right)_{A B}=\left(\nabla_{A} g\right)_{u B}=0$, which are readily seen to be contradictory.

Providentially enough, our definition for a conformal Carroll transformation does not involve the connection, though. Then a calculation analogous to the proof of Eq. (III.8) in the case (ii) shows that $L_{X} \mathrm{~g}=\lambda \mathrm{g}$ requires $\partial_{u} X^{A}=0$ as well as $L_{Y} \widehat{\mathrm{g}}=\left(\lambda-2 X^{u}\right) \widehat{\mathrm{g}}$. Using the second condition $L_{X} \xi=-(\lambda / N) \xi$ in (III.2) allows us to deduce that the conformal Carroll group of the punctured future light cone is (III.10), i.e., for $N=2$, the $B M S$ group. [18]

The condition $\lambda=0$ would fix the supertranslations as $T=X^{u}=-\frac{1}{2}\left(L_{Y} \widehat{\mathrm{g}}\right) / \widehat{\mathrm{g}}$, while leaving the space-part, $Y$, conformal; the Carroll "isometries" of the light-cone span therefore the conformal group, $\mathrm{O}(d+1,1)$, of the celestial sphere, $S^{d}$, with rigidly fixed "compensating" supertranslations.

\section{NEWMAN-UNTI GROUPS OF THE LIGHT-CONE}

Our formalism allows us to define the Newman-Unti group of a Carroll manifold, and of the light-cone in particular.
The Newman-Unti (NU) group is spanned of those (local) diffeomorphisms $a$ of $C$ which preserve the sole degenerate "metric", g, up to a conformal factor, namely $a^{*}[\mathrm{~g}]=[\mathrm{g}]$. This entails that the direction of $\xi$ is automatically preserved (since $a_{*} \xi$ lies again in the kernel of g). Its Lie algebra consists, hence, of all vector fields $X$ on $C$ such that $L_{X} \mathrm{~g}=\lambda \mathrm{g}$, the condition $L_{X} \xi=\mu \xi$ being automatically satisfied.

If $C$ is the light-cone $\mathcal{I}^{+}$of $\mathbb{R}^{d+1,1}$, we find that

$$
X=Y+X^{u}(x, u) \frac{\partial}{\partial u},
$$

with $Y=Y^{A}(x) \partial / \partial x^{A}$ a conformal vector field of $S^{d}$ and $X^{u} \in C^{\infty}(C, \mathbb{R})$, is, this time, an arbitrary function of the $x^{A}$ and $u$. The Newman-Unti group of the light-cone $C$ is, therefore,

$$
\mathrm{NU} \equiv \operatorname{Conf}\left(S^{d}\right) \ltimes C^{\infty}(C, \mathbb{R}),
$$

consistently with Eqs (I.5) and (I.6). We notice that Eq. (I.6) corresponds in fact to the "intermediate" Lie subalgebra of the NU Lie algebra defined by $L_{X} \mathrm{~g}=\lambda \mathrm{g}$, and $\left(L_{\xi}\right)^{2} X=$ 0 . For the light-cone, it consists in those vector fields $X=Y+X^{u} \partial / \partial u$ with, just as before, $Y \in \operatorname{conf}\left(S^{d}\right)$, and $\left(\partial_{u}\right)^{2} X^{u}=0$, i.e., such that $X^{u}=S[u+T]$, where $S$, and $T$ remain arbitrary functions on $S^{d}$, see Eq. (I.6).

Referring to Eqs (III.5) and (V.1) giving the generators of the conformal Carroll Lie algebras previously studied, we can highlight, 
in the case $N=2$, the interesting array of nested Lie groups [19]

$$
\mathrm{NU}_{1} \subset \mathrm{BMS} \subset \mathrm{NU}_{2} \subset \cdots \subset \mathrm{NU}
$$

We finally notice that $\mathrm{NU}_{1}=\mathrm{CCarr}_{\infty}$.

\section{CONCLUDING REMARKS}

The basic result of this paper is that the future null conformal boundary $\mathcal{I}^{+}$of an asymptotically flat spacetime emitting gravitational radiation is a Carroll manifold [7] and its asymptotic symmetries, i..e., elements of the Bondi-Metzner-Sachs group [1], constitute the associated conformal Carroll group. Originally introduced as the limit of the Poincaré group as the speed of light tends to zero [5], Carroll groups and Carroll manifolds have found applications in the study of velocity-dominated spacetimes and physics on branes which approach the speed of light. They are the analogue of Newton-Cartan manifolds, which arise when when the speed of light tends to infinity [7]. As a null hypersurface in the conformal compactification of spacetime $\mathcal{I}^{+}$carries an induced metric, g, which is degenerate with a one-dimensional kernel spanned by $\xi$ which is tangent to its null generators. It also carries a so-called strong conformal structure [10]. Depending upon how much of this structure one re- quires to be preserved one may obtain different symmetry groups. Dropping the requirement that the strong conformal structure be preserved leads to a larger group, namely to the Newman-Unti group [10], which is now seen to fit into the general theory of Carroll manifolds and their symmetries.

As we recalled in the introduction, there has been a considerable revival of interest recently in the BMS group in connection with its application to conformal field theory [2-4]. We hope that the clarification brought about in the present paper will further advance this study.

\section{Acknowledgments}

We are indebted to G. Barnich whose advice allowed us to correct an important misinterpretation. C.D. acknowledges ancient and enlightening discussions with F. Ziegler about the BMS group. GWG would like to thank KITP, Santa Barbara for its hospitality during its Bits and Branes program (2012), which provided a stimulus for this work. He is grateful also to the Laboratoire de Mathématiques et de Physique Théorique de l'Université de Tours for hospitality, and the Région Centre for a "Le Studium" research professorship. 
[1] H. Bondi, M. G. van der Burg, and A. W. Metzner, "Gravitational waves in general relativity. 7. Waves from axisymmetric isolated systems," Proc. Roy. Soc. Lond. A 269 (1962) 21; R. Sachs, "Asymptotic symmetries in gravitational theory," Phys. Rev. 128 (1962) 2851.

[2] G. Barnich and C. Troessaert, "Symmetries of asymptotically flat 4 dimensional spacetimes at null infinity revisited," Phys. Rev. Lett. 105 (2010) 111103 [arXiv:0909.2617 [gr-qc]]; “Aspects of the BMS/CFT correspondence JHEP 1005 (2010) 062 [arXiv:1001.1541 [hep-th]]; "BMS charge algebra," JHEP 1112 (2011) 105 [arXiv:1106.0213 [hep-th]]; G. Barnich, A. Gomberoff and H. A. Gonzalez, " $B M S_{3}$ invariant two dimensional field theories as flat limit of Liouville," Phys. Rev. D 87 124032, (2013) [arXiv:1210.0731 [hep-th]]; G. Barnich and P. -H. Lambert, "A note on the Newman-Unti group and the BMS charge algebra in terms of Newman-Penrose coefficients," J. Phys. Conf. Ser. 410 (2013) 012142; "Einstein-Yang-Mills theory : I. Asymptotic symmetries," Phys. Rev. D 88 (2013) 103006 [arXiv:1310.2698 [hep-th]].

[3] A. Bagchi, "Correspondence between Asymptotically Flat Spacetimes and Non- relativistic Conformal Field Theories," Phys. Rev. Lett. 105 (2010) 171601.

[4] A. Strominger, "Asymptotic Symmetries of Yang-Mills Theory," arXiv:1308.0589 [hep-th]; "On BMS Invariance of Gravitational Scattering," arXiv:1312.2229 [hepth]; T. He, V. Lysov, P. Mitra and A. Strominger, "BMS supertranslations and Weinberg's soft graviton theorem," arXiv:1401.7026 [hep-th].

[5] J. M. Lévy-Leblond, "Une nouvelle limite non-relativiste du group de Poincaré," Ann. Inst. H Poincaré 3 (1965) 1; V. D. Sen Gupta, "On an Analogue of the Galileo Group," Il Nuovo Cimento 54 (1966) 512. See [7] below and references listed in it.

[6] C. Duval, G.W. Gibbons, P. Horvathy, "Celestial mechanics, conformal structures and gravitational waves," Phys. Rev. D43 (1991) 3907 [hep-th/0512188].

[7] C. Duval, G. W. Gibbons, P. A. Horvathy and P. M. Zhang, "Carroll versus Newton and Galilei: two dual non-Einsteinian concepts of time," arXiv:1402.0657 [gr-qc].

[8] R. P. Geroch and E.T. Newman, "Application of the Semidirect Product of Groups," J. Math. Phys. 12 (1971) 314

[9] P. J. McCarthy, Structure of the BondiMetzner-Sachs Group J. Math. Phys. 13 
(1972) 1837-1842

[10] R. Penrose and W. Rindler, Spinors and Spacetime, Volume 2. Cambridge University Press (1986).

[11] M. A. Awada, G. W. Gibbons and W. T. Shaw, "Conformal Supergravity, Twistors And The Super BMS Group," Annals Phys. 171 (1986) 52.

[12] S. Hollands and A. Ishibashi, "Asymptotic flatness and Bondi energy in higher dimensional gravity," J. Math. Phys. 46 (2005) 022503 [gr-qc/0304054].

[13] C. Duval, and P. A. Horvathy "Nonrelativistic conformal symmetries and Newton-Cartan structures," J. Phys. A 42 (2009) 465206; [arXiv:0904.0531]. "Conformal Galilei groups, Veronese curves, and Newton-Hooke spacetimes," J. Phys. A 44 (2011) 335203 [arXiv:1104.1502].

[14] C. Duval, G. W. Gibbons and P. A. Horvathy, "Conformal Carroll groups," [arXiv:1403.4213 [hep-th].]

[15] A. O. Barut, "Conformal Group $\rightarrow$ Schrödinger Group $\rightarrow$ Dynamical Group - The Maximal Kinematical Group of the Massive Schrödinger Particle," Helv. Phys. Acta 46, 496 (1973); J. Negro, M. A. del Olmo, An A. Rodríguez-Marco, "Nonrelativistic conformal groups," J. Math. Phys. 38 (1997), 3786, and "Nonrelativistic conformal groups. II. Further devel- opments and physical applications," ibid. 3810; M. Henkel (2002) "Phenomenology of local scale invariance: from conformal invariance to dynamical scaling," Nucl. Phys. B 641, 405; J. Lukierski, P. C. Stichel, and W. J. Zakrzewski (2006) "Exotic Galilean conformal symmetry and its dynamical realizations," Phys. Lett. A 357, 1 [arXiv:hep-th/0511259]; A. Bagchi, and R. Gopakumar (2009) "Galilean Conformal Algebras and AdS/CFT," JHEP 0907, 037 [arXiv:0902.1385];

[16] The canonical $\nu$-densities of a Riemannian manifold $(\Sigma, \widehat{g})$ are locally of the form $f \operatorname{det}\left(\mathrm{g}_{A B}\right)^{\nu / 2}$ with $f$ a smooth function on $\Sigma$.

[17] The special value $z=1$ corresponds to group contraction from the relativistic conformal group, analogous to that in the Galilean case [15]. Accordingly, the conformal Carroll invariant g $\otimes \xi \otimes \xi$ can be regarded as a limit as $c \downarrow 0$ of the conformal invariant $c^{2} G \otimes G^{-1}$ of a Lorentz manifold $(M, G)$ for which the Carroll manifold is an ultra-relativistic asymptote.

[18] As explained before, for $d=1$ and $N=2$ the conformal Carroll group is the same as CGA with $z=1$, as seen by interchanging position and time, cf. [3].

[19] The $X^{u}$ component of the generators (V.1) is a polynomial of degree $k-1$ in $u$. 